\title{
Optimal Power Allocation Scheme for Downlink CoMP Systems
}

\author{
Jun $\mathrm{Li}^{1}$, Han Hai ${ }^{1}$, Ying $\mathrm{Guo}^{2}$, Moon Ho Lee ${ }^{1}$ \\ ${ }^{1}$ Department of Electronic and Information Engineering, Chonbuk National University, Jeonju, Korea \\ ${ }^{2}$ School of Information Science \& Engineering Central South University, Changsha, China \\ Email: lijun52018@jbnu.ac.kr, hhhtgy@jbnu.ac.kr,yingguo@mail.csu.edu.cn,moonho@jbnu.ac.kr
}

Received 2013

\begin{abstract}
Coordinated multi-point transmission and reception (CoMP) scheme enable LTE-Advanced systems to achieve their higher spectral efficiency. Allowing base stations to cooperate one another is one of the solutions to mitigate the intercell interference (ICI). In this paper, we propose an iterative power allocation scheme with MMSE procoding based on a modified water-filling for downlink CoMP systems, which achieves the optimal performance. The simulation results show that our proposed system can achieve its optimal rate according to its antenna configuration. Comparing them with a block diagonalization (BD) shows the advantages of MMSE precoding, in particular at a low SNR region.
\end{abstract}

Keywords: CoMP; Precoding; Power Allocation; Waterfilling

\section{Introduction}

Coordinated multi-point transmission and reception (CoMP) scheme has been widely used for LTE-Advanced system to enhance cell average and cell edge throughput. According to a coordinating fashion, CoMP scheme is classified into two strategies [1]: Joint processing (JP) and coordinated scheduing/beamforming (CS/CB). In the JP strategy as shown in Figure 1, each user equipment (UE) simultaneously receives data from multiple base stations (BSs) with joint multi-user precoding, which is required to share both user data and channel state information (CSI) between base stations (BSs) and user equipments (UEs). Stemming from that, this JP strategy could be used to contribute to not only improving the strength of the receive signal but also cancelling interference. However, it is required to exchange the significant amount of data among links participating in its coordination. Besides, substantial overheads should be taken into account in the network.

Compared with JP, CS/CB can avoid inter-cell interference (ICI) by applying precoding to each BS on an individual basis, which is required to share only CSI without holding user data in common. As depicted in Figure 2, the solid lines denote the desired signals and the dashed lines denote the interference from other BSs locating in other cells. Since sharing CSI requires much lower capacity than sharing data [2], CS/CB needs much lower backhaul capacity than JP. A lot of studies have been done on CS/CB [3-5]. However, an effective algorithm to eliminate the interference for downlink CoMP and network MIMO is still not sufficient. If the interference is known at the transmitters, cooperative encoding using dirty paper coding (DPC) could mitigate the inter-cell interference (ICI) [6]. Apart from DPC, a zero-forcing (ZF) scheme based on block diagonalization (BD) is proposed in [7]. In [8], BD is applied to a multi-cell scenario with an ICI reduction scheme. However, as pointed out in [9], performance of BD is suboptimal at a low SNR. In this paper, we use the MMSE precoding to compensate the noise with the interference, with the goal of maximizing the weighted sum rate (WSR).

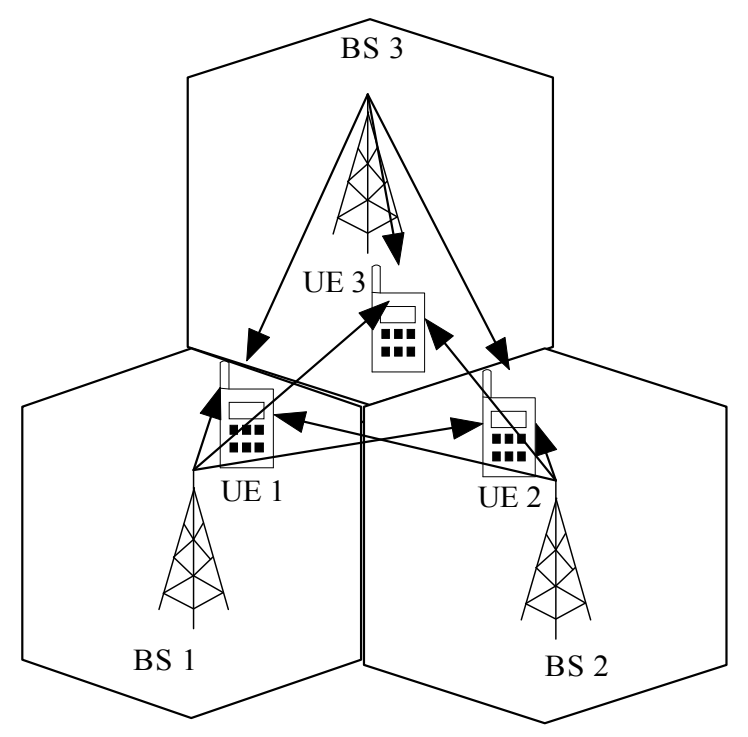

Figure 1. Joint processing (JP). 


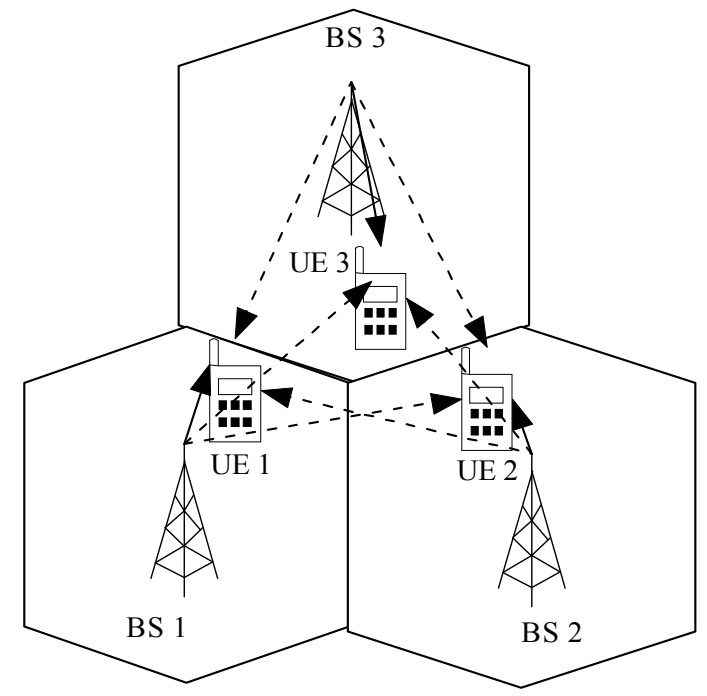

Figure 2. Coordinated scheding/beamforming (CS/CB).

A solution to maximizing the WSR is proposed in [10] on the condition that each UE is equipped with a single antenna and the precoding matrix is chosen in order to maximize the signal-noise-plus-interference ratios (SINR) for each user. Aside from this, a power allocation scheme is also proposed. In [11], non-iterative water-filling scheme is proposed to solve the problem occurring when applying BD.

This paper is organized as follows. In Section 2, we show a proposed system model. In Section 3, we overview conventional power allocation schemes and propose a modified optimal power selection scheme. In Section 4, we show that our proposed system outperforms conventional systems using computer simulation. In Section 5, we come to some conclusions.

\section{System Model}

Consider a cooperative cluster which is comprised of both $\mathrm{N} \mathrm{BSs}$ and N UEs on the condition that each BS is equipped with $\mathrm{N}_{t}$ antennas while each UE is equipped with a single antenna. In addition, each cell has both a single BS and UE, under the situation that each UE regards the nearest $\mathrm{BS}$ as its local BS.

Figure 3 illustrates our system model. Each BS is denoted by each circle, which is represented by the (i), where $1 \leq \mathrm{i} \leq \mathrm{N}$. Aside from this, $\mathbf{h}_{j, i} \in \mathbb{C}^{\mathrm{N}_{t} \times 1}$ indicates the channel vector from a coordinated $\mathrm{BS}_{\mathrm{j}}$ to a $\mathrm{UE}_{\mathrm{i}}$, while $\mathbf{v}_{i}=\left[\mathrm{v}_{i}^{1}, \ldots, \mathrm{v}_{i}^{\mathrm{N}_{t}}\right]$ indicates a beamforming vector used to mitigate the interference between $\mathrm{BS}_{\mathrm{i}}$ and $\mathrm{UE}_{\mathrm{i}}$. In addition, the solid lines denote the desired signals while dashed lines denote the interference from the coordinated BSs.

Our proposed system is represented as follow. The received signal of $\mathrm{UE}_{\mathrm{i}}, \quad y_{i}$ is given by $\mathrm{BS}_{\mathrm{i}}$ with power $P_{i}$

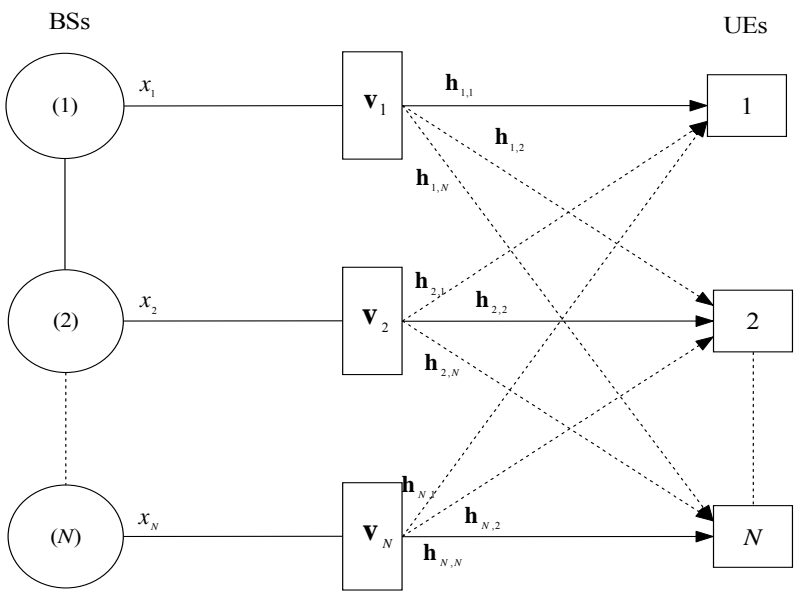

Figure 3. Downlink Multi-user beamforming of the CoMP.

under CoMP transmission mode where $i=1, \ldots, N$,

$$
y_{i}=\mathbf{h}_{i, i}^{H} \mathbf{v}_{i} X_{i}+\sum_{j=1, j \neq i}^{N} \mathbf{h}_{j, i}^{H} \mathbf{v}_{j} x_{j}+z_{i} .
$$

Besides, $x_{j}$ is the inter-cell interference signal transmitted by $\mathrm{BS}_{\mathrm{j}}$. The desired signal $x_{i}$ of $\mathrm{UE}_{\mathrm{i}}$ is only transmitted by $\mathrm{BS}_{\mathrm{i}} . \mathrm{z}_{u}$ denotes the noise at $\mathrm{UE}_{\mathrm{i}}$, which is a white Gaussian random variable with zero mean and variance $\sigma^{2}$

\section{Power Allocation Scheme}

The design of the precoding matrix $\mathbf{V}=\left[\mathbf{v}_{1}, \cdots \mathbf{v}_{i}, \cdots, \mathbf{v}_{N}\right]$ and the optimization of the powers $P_{i}$ are chosen in order to maximize a WSR:

$$
R=\sum_{i=1}^{N} \alpha_{i} \log _{2}\left(1+\operatorname{SINR}_{i}\right)
$$

where $S I N R_{i}$ is the signal to noise-plus-interference ratio at each receive antenna. The value $\alpha_{i} \in[0,1]$ indicates the priority of each user, in particular case of equal priority, $\alpha_{i}=1 / N$, for all $i$.

In this section, the received SINR for UEi is

$$
i \operatorname{SINR}_{i}=\frac{\left|\mathbf{h}_{i, i}^{H} \mathbf{v}_{i}\right|^{2} P_{i}}{\sigma^{2}+\sum_{j=1, j \neq i}^{N}\left|\mathbf{h}_{j, i}^{H} \mathbf{v}_{j}\right|^{2} P_{j}},
$$

where $|$.$| represents the norm, \left|\mathbf{h}_{i, i}^{H} \mathbf{v}_{i}\right|^{2}$ is the desired signal power of UEi, and $\sum_{j=1, j \neq i}^{N}\left|\mathbf{h}_{j, i}^{H} \mathbf{v}_{j}\right|^{2} P_{j}$ indicates the interference signal power from the other BSs. It should satisfy the condition of power constraint $P_{\max B S}$ :

$$
\sum_{i=1}^{N} P_{i}\left|\mathbf{v}_{i}\right|^{2} \leq P_{\max B S}
$$


The beamforming matrix $\mathbf{V}$ is chosen according to MMSE criterion [12], which maximizes the SINR at the receiver. Therefore, the beamforming matrix is represented as

$$
\mathbf{V}=\mathbf{H}^{H}\left(\mathbf{H H}^{H}+\frac{1}{\rho} \mathbf{I}_{N}\right)^{-1},
$$

where $\mathbf{H}=\left[\mathbf{h}_{1} \ldots \mathbf{h}_{i} \ldots \mathbf{h}_{M}\right]^{T}, \quad \rho$ is the signal-to-noise ratio (SNR), defined as a ratio of a possible maximum power and noise power $\sigma^{2} . \mathbf{I}_{N}$ is an $\mathrm{N}$ by $\mathrm{N}$ identity matrix. isfy

The general problem is to find the powers $P_{i}$ to sat-

$$
\max \left\{\sum_{i=1}^{N} \alpha_{i} \log _{2}\left(1+\operatorname{SINR}_{i}\right)\right\},
$$

where $\alpha_{i} \in[0,1]\left(\sum_{i=1}^{N} \alpha_{i}=1\right)$.

For simplicity without loss of generality, we can approximate the equation (6) as

$$
\sum_{i=1}^{N} \alpha_{i} \log _{2}\left(1+\frac{\left|\mathbf{h}_{i, i}^{H} \mathbf{v}_{i}\right|^{2} P_{i}}{\sum_{j=1, j \neq i}^{N}\left|\mathbf{h}_{j, i}^{H} \mathbf{v}_{j}\right|^{2} P_{j}}\right) .
$$

Since the powers of different users are coupled, we set an optimization approach. We assume that all the powers except $P_{i}$ are set, that is $P_{j}, j \neq i$. Therefore, two suboptimum procedures are proposed to derive a closedform solution.

\subsection{Waterfilling Scheme}

The power allocation of waterfilling scheme is given by

$$
p_{i}=\left[K \alpha_{i}-\frac{\sum_{i \neq j}\left|\mathbf{h}_{j, i}^{H} \mathbf{v}_{j}\right|^{2} P_{j}+\sigma^{2}}{\left|\mathbf{h}_{i, i}^{H} \mathbf{v}_{i}\right|^{2}}\right]^{+},
$$

where $[\cdot]^{+}$denotes the maximum between zero and the argument. The constant $\mathrm{K}$ can be found in [12]. In particular, $\alpha_{i}=1 / N$ is chosen for a standard waterfilling.

\subsection{Modified Waterfilling Scheme}

The maximum sum of squared weights is defined as:

$$
\Omega_{i}^{B S}=\max _{k=1, \ldots, N}\left(\left|\mathbf{v}_{k}\right|^{2}\right) .
$$

Therefore, we have to find a constant value $\mathrm{K}$ for all the power $P_{i}$, the following equation holds:

$$
p_{i}=\left[K \frac{\alpha_{i}}{\Omega_{i}^{B S}}-\frac{\sum \sum_{i \neq j}\left|\mathbf{h}_{j, i}^{H} \mathbf{v}_{j}\right|^{2} P_{j}+\sigma^{2}}{\left|\mathbf{h}_{i, i}^{H} \mathbf{v}_{i}\right|^{2}}\right]^{+} .
$$

This corresponds to a watrefilling distribution with variable water level.

In block diagonalization, the SINR is given by [13]

$$
\operatorname{SINR}_{i}=\frac{\left|\lambda_{i}\right|^{2} P_{i}}{\sigma^{2}},
$$

where $\lambda_{i}$ are the diagonal elements of $\mathbf{H V}$ where the precoding matrix is chosen from [13]. Therefore, the weighted sum rate is

$$
R=\sum_{i=1}^{N} \alpha_{i} \log _{2}\left(1+\frac{\left|\lambda_{i}\right|^{2} P_{i}}{\sigma^{2}}\right) .
$$

The solution is given as [11]:

$$
P_{i}=\left[K \alpha_{i}-\frac{\sigma^{2}}{\left|\lambda_{i}\right|^{2}}\right]^{+},
$$

which is also a waterfilling distribution with equal priority.

In fact, the procedures of iterative optimization as presented before are based on the fact that the powers $P_{j}$ for $j \neq i$ are known to obtain $P_{i}$. For this, a joint optimization should be found. Therefore, we should repeat the aforementioned waterfilling procedures to find each $P_{i}$ by adjusting $P_{j}$ values of the preceding step until the resulting rate converges after setting arbitrary initial values for $P_{j}$.

\section{Simulation Results}

In this section, we analyze the performance in terms of achievable rates for the proposed iterative waterfilling (MMSE PWF), modified waterfilling (MMSE PMWF) and the optimum solution found by exhaustive search (MMSE ES). We also compare these performances to those of block-diagonalization using a waterfilling scheme (BD WF).

For these numerical results, the channel matrix entries are assumed to be independent identically distributed zeromean complex Gaussian random variables with variance of 0.5 per dimension. The initial powers for the iterative WF and MWF have been set equal to the $P_{i}$ of the uniform power distribution and the final power allocations are obtained after 5 iterations in all the simulations. For a fair comparison, SNR is defined as the ratio in $\mathrm{dB}$ of $P_{\max B S} / \sigma^{2}$.

In Figure 4, we compare the different solutions in terms of mean achievable rate for each user on the condition that each user has the same priority and the SNR is set to $0 \mathrm{~dB}$. We can see that the optimum MMSE precoding found by ES outperforms BD WF for all the values of the number of transmit antennas $N_{t}$, and MMSE PMWF also outperforms MMSE PWF. Interestingly, the simple solution of MMSE PMWF outperforms BD WF 
that requires a lengthy numerical optimization, in particular at the low SNR.

In addition, we obtain the region of achievable rates for $\mathrm{SNR}=0 \mathrm{~dB}$ in Figure 5, where we show the effect of the different power allocation schemes with MMSE precoding. We can see that MMSE PMWF obtains higher achievable rates than MMSE PWF. As the number of transmit antennas increases, the difference between MMSE ES and MMSE MWF is larger. However, even if suboptimal, MMSE MWF outperforms BD WF as shown in Figure 1, without resorting to a lengthy numerical optimization.

The main difference in terms of complexity between MMSE and BD approaches is coming from the power optimization procedure. From the simulation results, we can see that the optimum power distribution can be obtained through a lengthy exhaustive search while waterfilling approaches allow a highly reduced complexity at

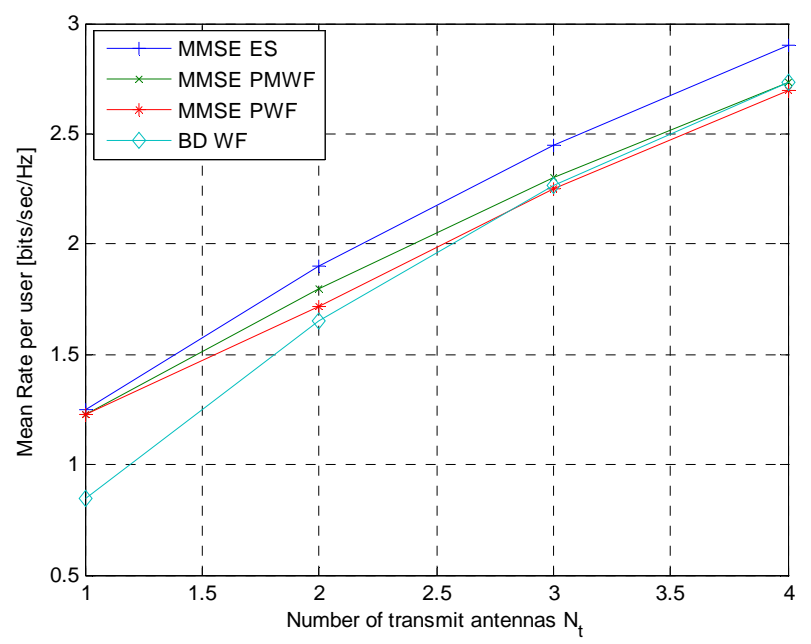

Figure 4. Mean achievable rate for each user with equal probability, $N=2$ and $S N R=0 \mathrm{~dB}$.

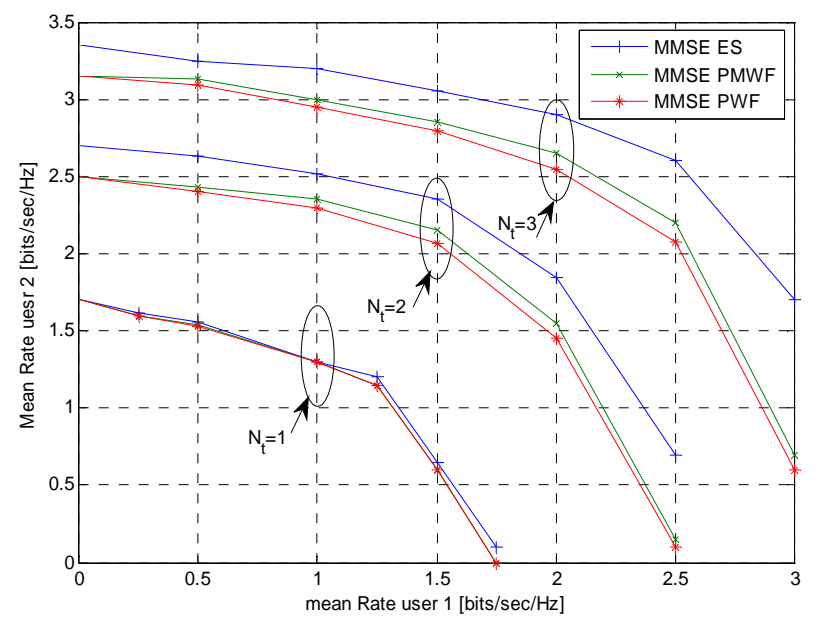

Figure 5. Region of mean achievable rates with $N=2$ and $S N R=0 \mathrm{~dB}$. the expense of some performance degradation. Since waterfilling is performed over $\mathrm{N}$ user transmissions, it does not depend on the number of transmit antennas. Therefore, the complexity of PWF and PMWF is irrespective of the number of antennas per BS. In addition, MMSE PMWF is a good choice in terms of the balance between complexity and achievable rates.

\section{Conclusions}

In this paper, we present feasible combinations of MMSEbased beamforming and iterative waterfilling power allocation schemes that can be applied to the downlink of a CBST system while they are required to take a tradeoff between complexity and achievable rates into consideration. In addition, we show that the iterative MWF allocation combined with MMSE beamforming outperforms a $\mathrm{BD}$ scheme that requires a lengthy numerical optimization in the low and moderate SNR regimes under a two-base station/two-user simplified scenario. Furthermore, we show that our proposed iterative MWF scheme obtains a performance close to that of MMSE by exhaustive search at a high SNR regime as well as MMSE and $\mathrm{BD}$ have the same performance at a high SNR regime.

\section{Acknowledgements}

This work was supported by the National Research Foundation of Korea (NRF) grant funded by the World Class University R32-2012-000-20014-0, the Korean government (MEST) 2012-002521, and BSRP 20100020942 Korea.

\section{REFERENCES}

[1] M. Sawahashi, Y. Kishiyama, A. Morimoto, M. Nishikawa and D. Tanno, "Coordinated Multipoint Transmission/Reception Techniques for Ite-advanced," IEEE Wireless Communications, Vol. 17, No. 3, 2010, pp. 26-34. doi.org/10.1109/MWC.2010.5490976

[2] D. Samardzija and H. Huang, "Determining Backhaul Bandwidth Requirements for Network MIMO," $17^{\text {th }}$ European Signal Processing Conference (EUSIPCO 2009), Glasgow, Scotland, 2009, pp. 1494-1498.

[3] W. Choi and J. G. Andrews, "The Capacity Gain from Intercell Scheduling in Multi-antenna Systems," IEEE Transactions on Wireless Communication, Vol. 7, No. 2, 2008, pp. 714-725. doi:10.1109/TWC.2008.060615

[4] T. Ren and R. J. La, "Downlink Beamforming Algorithms with Intercell Interference in Cellular Networks," IEEE Transactions on Wireles Communincation, Vol. 5, No.10,2006,pp.2814-2823.doi:10.1109/TWC.2006.04580

[5] S. G. Kiani and D. Gesbert, "Optimal and Distributed Scheduling for Multicell Capacity Maximization," IEEE Transactions on Wireless Communication, Vol. 7, No. 1, 2008, pp. 288-297. doi:10.1109/TWC.2008.060503 
[6] S. Shamai (Shitz) and B. M. Zaidel, "Enhancing the Cellular Downlink Capacity via Co-processing at the Transmitting End," IEEE Vehicular Technology Conference (VTC2001-Spring), Rhodes, Greece, 2001, pp. 17451749.

[7] Q. H. Spencer, A. L. Swindlehurst and M. Haardt, "Zero-Forcing Methods for Downlink Spatial Multiplexing in Multiuser MIMO Channels," IEEE Transactions on Signal Processing, Vol. 52, No. 2, 2004, pp. 461-471. doi:10.1109/TSP.2003.821107

[8] J. Zhang, R. Chen, J. G. Andrews, A. Ghosh, and R. W. Heath, "Networked MIMO with Clustered Linear Precoding," IEEE Transactions on Wireless Communications, Vol. 8, No. 4, 2009, pp. 1910-1921. doi:10.1109/TWC.2009.080180

[9] E. Bjornson, R. Zakhour, D. Gesbert and B. Ottersten, "Distributed Multicell and Multiantenna Precoding: Characterization and Performance Evaluation," IEEE Global Communications Conference (GLOBECOM 2009), Hawaii, U.S.A, 2009, pp. 1-6.
[10] R. S. Cheng and S. Verdu, "Gaussian Multiaccess Channels with ISI: Capacity Region and Multiuser Water-filling," IEEE Transactions on Information Theory, Vol. 39, No. 3, 1993, pp. 773-785. doi: $10.1109 / 18.256487$

[11] A. Garcia Armada, M. Sanchez Fernandez and R. Corvaja, "Waterfilling Schemes for Zero-Forcing Coordinated Base Station Transmission," IEEE Global Communications Conference (GLOBECOM 2009), Hawaii, U.S.A, 2009.

[12] J. M. Cioffi, "Advanced Digital Communications, EE379c," Stanford University Course Notes. http://www.stanford.edu/class/ee379c.

[13] G. J. Foschini, K. Karakayali and R. A. Valenzuela, "Coordinating Multiple Antenna Cellular Networks to Achieve Enormous Spectral Efficiency," IEEE Proceedings Communications, Vol. 153, No. 4, 2006, pp. 548-555. 